\title{
Eine Grabplatte in der Urbanskirche und ihre Geschichte
}

\author{
von HeLMut WÖRNER
}

\section{Die Grabplatte}

In der Urbanskirche in Schwäbisch Hall-Unterlimpurg wurde bei Renovierungsarbeiten eine Grabplatte mit Limpurger Wappen im wahrsten Sinne des Wortes ent-deckt.

Sie liegt im Boden des Chors links neben dem Altar, unterhalb des Sakramentshauses. Auf ihr stand vermutlich eine sehr lange Zeit ein kleines Chorgestühl. Genauer gesagt, stand das Gestühl auf der halben Grabplatte, die dadurch geschützt war. Die andere Hälfte dagegen ist vollkommen abgetreten und glatt. Dort ist nichts mehr von der Umschrift oder dem Wappen zu erkennen. Fast sieht es so aus, als ob dieser Teil der Grabplatte bearbeitet worden wäre, um ihn dem Niveau des umgebenden Chorbodens anzugleichen.

Bei der Renovierung wurde das Chorgestühl entfernt und so der Stein freigelegt. Erhalten ist in der Mitte die Hälfte des Wappens der Schenken von Limpurg. Ins Auge springt auch die Jahreszahl 1530. Der erhaltene Teil der Umschrift lässt sich wie folgt entziffern:

[d]om 1530 / [a]m sampstag vorm balmtag starb der wol / geborn got [t]

Der Text bricht mitten im Namen des Verstorbenen ab. Doch in Verbindung mit der Jahreszahl drängt sich die Vermutung auf, dass es sich hier um die Grabplatte Schenk Gottfried von Limpurgs handeln könnte. Dafür spricht eine Reihe von Indizien.

Der Stein unterhalb des Sakramentshauses, das möglicherweise von Gottfrieds Urgroßmutter Elisabeth von Hohenlohe gestiftet worden war, befindet sich an einem besonders hervorgehobenen, ehrenvollen und heilbringenden Begräbnisplatz, was für die Bestattung einer hochgestellten Persönlichkeit spricht.

Schenk Gottfried (1474-1530) ist der einzige der regierenden Schenken von Limpurg vom 14. Jahrhundert bis zur Mitte des 16. Jahrhunderts, der nicht im Erbbegräbnis der Limpurger auf der Comburg beigesetzt wurde und dort auch nicht mit einem Epitaph oder Grabstein vertreten ist. Sein Sohn Erasmus war 1553 der letzte Schenk von Limpurg, der, wiewohl schon evangelisch, sich noch auf der Comburg beisetzen ließ. Die Schenken von Limpurg hatten danach in der Obersontheimer Kirche ihren Begräbnisplatz, so wie schon zuvor die Gaildorfer Linie in der Stadtkirche in Gaildorf. 


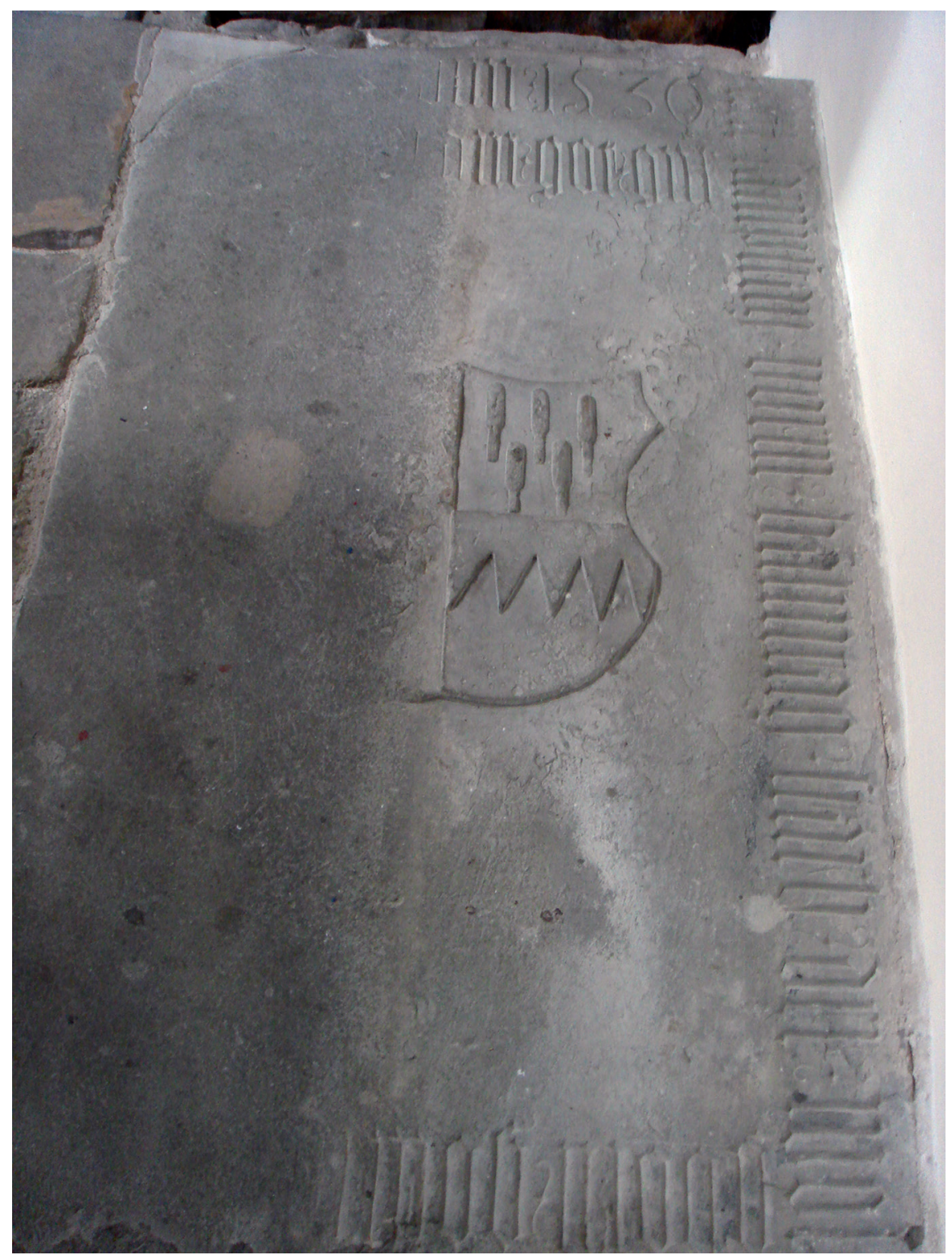

Abb. 1 Grabplatte unter dem Sakramentshaus der Urbanskirche (Aufnahme: Helmut Wörner) 
Schenk Gottfried hatte ein sehr gespanntes Verhältnis zum Stift Comburg. Bekannt ist das Wort von den „Gottsböswichtern“ der Comburg, die das Dach über der Schenkenkapelle zerfallen und es auf die Epitaphe hereinregnen ließen ${ }^{1}$. Christoph Fröschel beschreibt in seiner Limpurger Chronik auf 51 Seiten das Leben Schenk Gottfrieds. Das Kapitel darin über seine Beziehung zu Comburg beginnt so: Was es der wolgedachter Herr Schenk Gottfrid für eine Nachbarschaft mit dem Stift Camberg gehabt, wie unfreindlich und widerspenstig sie sich erzeigt haben, were hiehero zu lang zu erzelen ${ }^{2}$. Und dann folgen 45 Seiten, die allein dem nicht enden wollenden Streit zwischen Gottfried und den Stiftsherren von Comburg über die Schirmpflicht und die Verleihung der Pfründen gewidmet sind. Dass er unter solchen Umständen nicht auf der Comburg begraben werden wollte oder konnte, liegt nahe.

Doch es gibt auch eine Reihe schriftlicher Quellen zum Begräbnisort Schenk Gottfrieds.

Heinrich Prescher berichtet kurz: Gottfried von Limpurg, $\dagger 1530$, Samstag vorm Palmtag, in der Kirche zu Unterlimpurg begraben. ${ }^{3}$

Ganz ähnlich heißt es bei Gottfried Biedermann": Gottfried II. [...], Herr zu Limpurg, starb Anno 1530 Samstags vor Palmarum, und liegt in der Kirche unterhalb Limpurg, bey Halle begraben.

Christoph Fröschel, der seine Limpurger Chronik Ende des 16. Jahrhunderts verfasste, und Georg Salomon Ziegler, der seine Beschreibung des Limpurger Landes und der Limpurger Geschichte, den so genannten Limpurger Ehrensaal, 1739 fertigte, gehen beide ausführlich auf den Beisetzungsort und den Grabstein Schenk Gottfrieds ein.

Fröschel schreibt: Anno 1530 am Sambstag vorm Palmabend zwischen fünf und sechs Uhr ist Herr Schenk Gottfried zu Limpurg gestorben. Liegt begraben im Chor des Kirchleins herund unter Limpurg neben dem Altar unter einem Grabstein, darauf nur schlechtlich der Limpurgische Schild gehauen ohn ein Helm, an den vier Orten herum stehet diese Schrift:

Anno Domini 1530 am Sambstag vor Palmtag starb der wolgeborn Gottfrid Herr zu Limpurg des Römischen Reich Erbschenk und Semperfrey [...] dem Gott gnedig sey ${ }^{5}$.

Unter den in einem anderen Band überlieferten Zeichnungen der Grabmäler von Mitgliedern des Hauses Limpurg ${ }^{6}$ von 1593 findet sich auch der Grabstein

1 Christoph Fröschel: Das uralte Herkommen, Stamm und Geschlecht der Herrn zu Limpurg [...], 1593. Abschrift um 1600. S. 280 (StA Ludwigsburg B 113 I Bü. 2197).

2 Ebd., S. 262.

3 Heinrich Prescher: Geschichte und Beschreibung der zum fränkischen Kreise gehörigen Reichsgrafschaft Limpurg. 2. Teil. Stuttgart 1790.

4 Johann Gottfried Biedermann: Genealogie der Hohen Grafenhäuser im Fränckischen Crayse. 1. Teil. Erlangen 1745. Tabula XCI.

5 Wie Anm. 1, S. 307.

6 Zeichnungen der Grabmäler von Mitgliedern des Hauses Limpurg..., 1593 (StA Ludwigsburg B 113 I Bü 2198). 


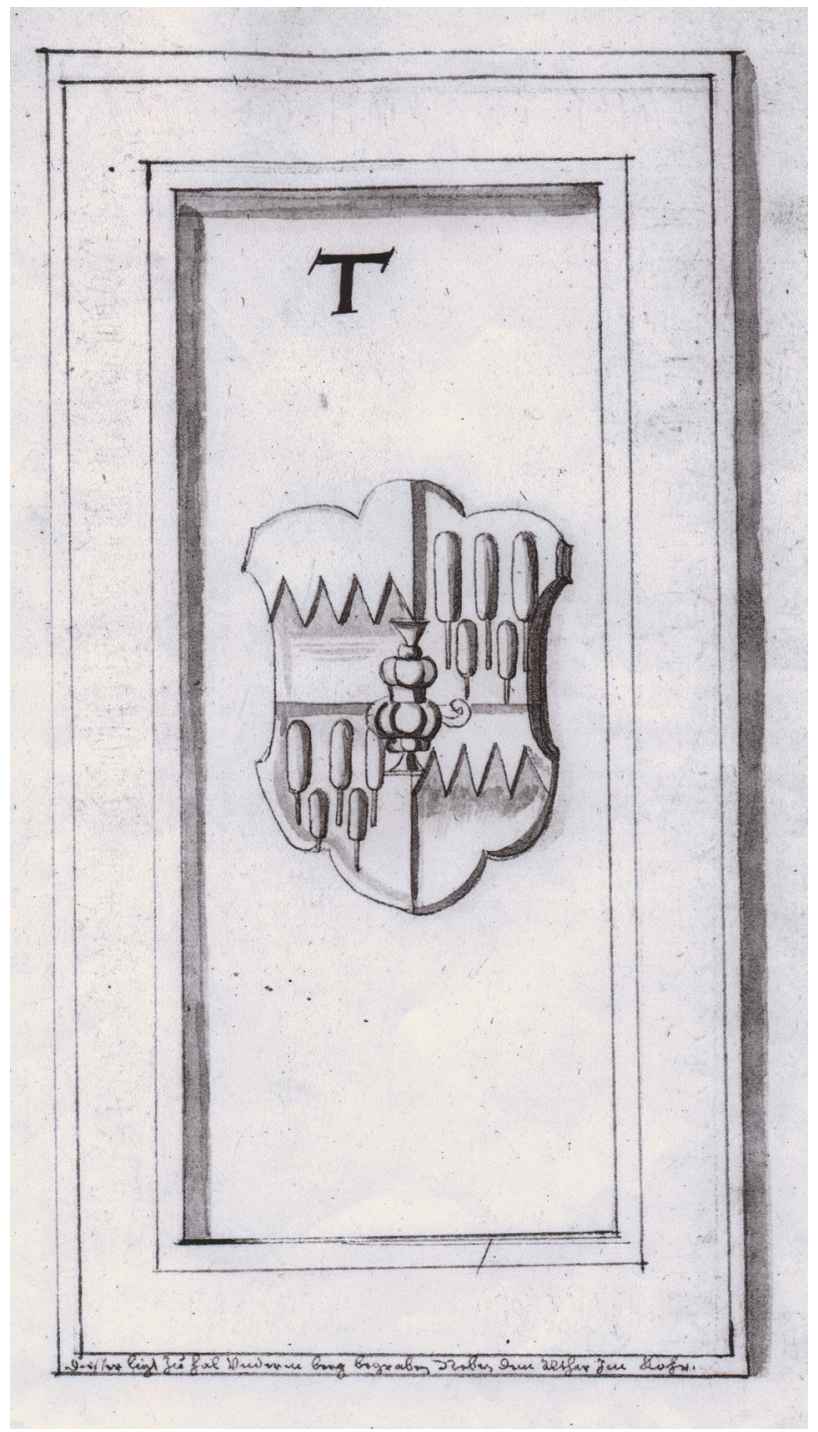

Abb. 2 Lavierte Tuschezeichnung der Grabplatte Schenk Gottfrieds in der Schwäbisch Haller Urbanskirche, 1593 (Aufnahme: Staatsarchiv Ludwigsburg)

Schenk Gottfrieds (bezeichnet mit dem Buchstaben T) mit dem Vermerk: Diesser ligt zu Hal Vnderm berg begraben Neben dem Althar Im Kohr. Die Inschrift des Grabsteins ist in Christoph Fröschels Chronik überliefert (vgl. Abb. 2 und 3). 


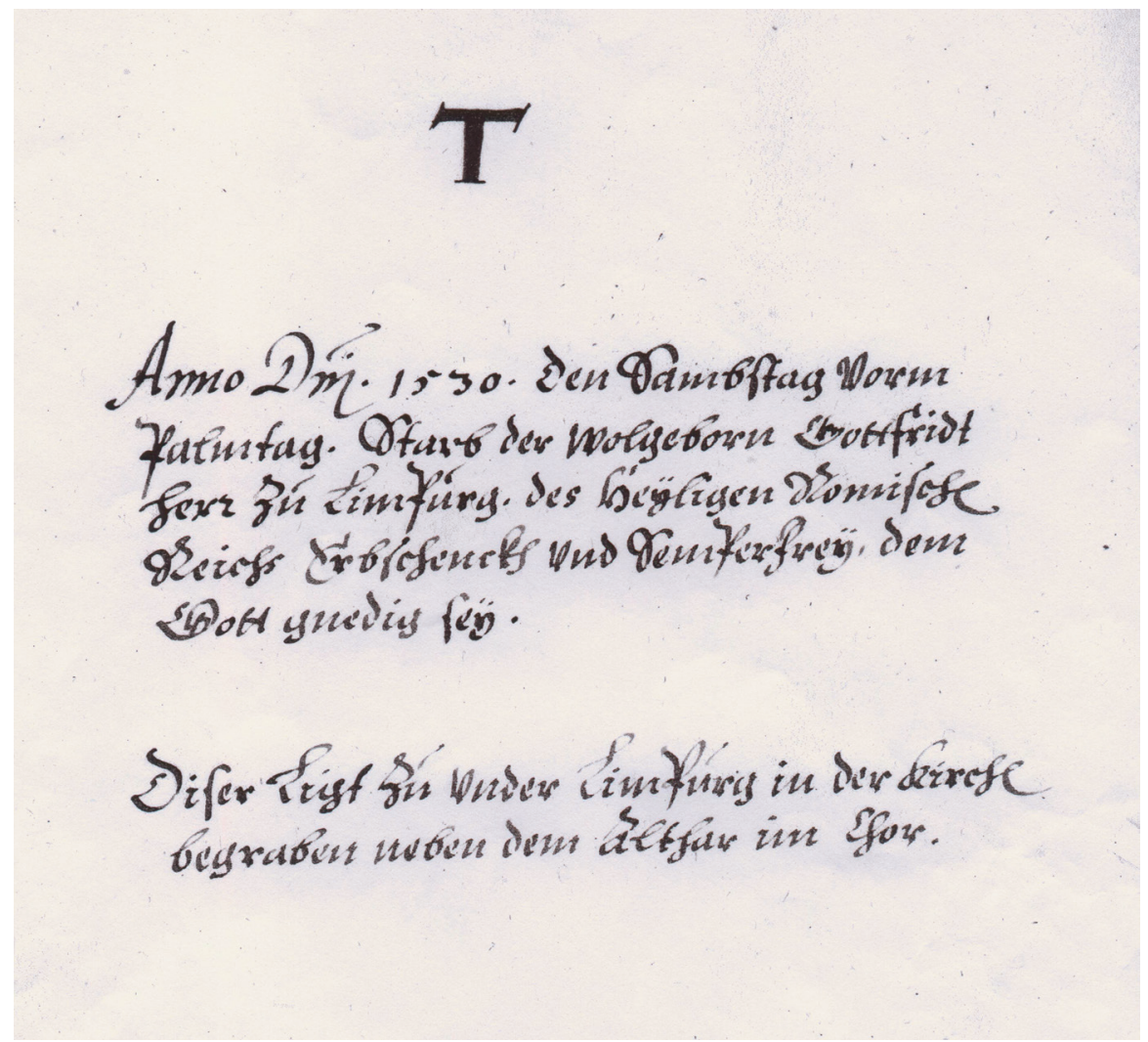

Abb. 3 Text zum Grabstein Schenk Gottfrieds (Aufnahme: Staatsarchiv Ludwigsburg)

Georg Salomon Ziegler wird die Chronik Fröschels als Quelle benutzt haben. Doch über die reine Beschreibung hinaus macht er sich auch Gedanken, warum wohl Schenk Gottfried nicht auf der Comburg beigesetzt wurde:

Sch[enk] Gottfrids verblichener Leichnam wurde in dem Kirchlein zu Unter Limpurg, unterhalb seines Residenz-Schloßes Limpurg, in dem Chor neben dem Altar, beygesetzt. Sein Grab siehet man bedeckt mit einem Stein, auff welchem der Limpurgische Stammes-Wappen-Schild ohne Helm, blo $\beta$ allein, ausgehauen, wahrgenom (m)en wird. Der Rand um diesen Stein herum hält nachgesetzte Nachricht-Schrifft in sich:

Anno Domini 1530 am Samstag vor PalmTag / starb der wolgeborn Gottfried Her zu Limpurg / des Röm[ischen] Reichs Erbschenck und Semperfrey / dem Gott gnädig sey 
Es ist merckwürdig, daß, weil sonst alle Herren von Limpurg, welche im Schloß Limpurg bey Hall mit Tod abgegangen, in ihrem eigenen Erbbegräbniß in der Schencken-Capelle an der Stiffts-Kirche zu Comberg begraben worden, gleichwol bemeldten Sch[enk] Gottfrids Leichnam nicht dahin gebracht worden: und wird sonder Zweiffel Ursache seyn, entweder, daß Sch[enk] Gottfrid oder die Seinigen aus Verdruß über dem, was Ihnen bißher Unbilliges von dem Stifft Comberg begegnet, ein anders mit seiner Begräbni $\beta$ verordnet, also daß Er todt daselbst, wo Er, noch lebend, als Schirm-Herr nicht gern wollen gesehen werden, nicht seyn wollen noch sollen: oder, daß das Stifft Comberg seinen Leichnam in die bemeldte Capelle einzulaßen sich gewidert, dahero man, Schmach und Weitläufigkeiten zu vermeiden, lieber das nächste ergriffen, und den Leichnam Schenck Gottfrids aus seinem Hof-Sitz Limpurg in erwähntes Kirchlein zu Unter Limpurg, ohne sondere Mühe und Ungelegenheit zur Ruhe brachte, und standsmäßig solchen bestattigte ${ }^{7}$.

Damit dürfte gesichert sein, dass sich nicht nur die Grabplatte, sondern auch das Grab Schenk Gottfried von Limpurgs in seiner Herrschaftskirche unter der Limpurg, der heutigen Urbanskirche, befindet.

Vermutlich war dieses bald vergessen. Das lässt sich leicht nachvollziehen, wenn man bedenkt, dass nur elf Jahre nach dem Tod Schenk Gottfrieds sein Sohn Erasmus die Burg Limpurg mitsamt dem Ort Unterlimpurg und seiner Kirche an die Reichsstadt Hall verkaufte und nach Obersontheim zog. Die Haller Bürger hatten wohl keinen besonderen Respekt vor einer Grabplatte eines Schenken oder scherten sich einfach nicht darum. So wurde irgendwann, um jeden Platz in dem Kirchlein zu nutzen, auf diese ein zusätzliches Chorgestühl gestellt. Die offenliegenden, als Relief ausgearbeiteten Buchstaben der Umschrift und Teile des mittigen Wappens sind vielleicht zur besseren Begehbarkeit der Platte gleich abgespitzt oder von den Kirchstuhlbenutzern im Lauf der Zeit abgetreten worden.

\section{Schenk Gottfried II. von Limpurg}

Schenk Gottfried II. wurde am 1. Juni 1474 als jüngster Sohn von Schenk Georg und Margarethe von Hohenberg geboren. Sein Vater wird für uns verkörpert durch den gerüsteten Ritter, dessen eindrucksvolles Grabdenkmal im Kapitelsaal der Comburg sofort ins Auge springt. Schenk Georg starb, noch nicht 40 Jahre alt, vermutlich während des Feldzuges gegen den Herzog Karl von Burgund bei Neuß. Die Streitigkeiten mit der Reichsstadt Hall hatten sich in den 1470er Jahren immer mehr zugespitzt, und so wurde als Ursache für seinen plötzlichen Tod am 10. Mai 1475 in der Überlieferung auch eine Vergiftung durch die Haller

7 Georg Salomon Ziegler: Limpurgischer Ehrensaal (StA Würzburg, Manuskript-Sammlung 166 II). 
nicht ausgeschlossen. Die Großmutter Schenk Gottfrieds ist die heute noch im Haller Land bekannte und fast schon sagenumwobene Susanna von Thierstein. Und sein Namenspatron war Gottfried, der Bruder seines Großvaters Friedrich V., der von 1444 bis zu seinem Tod 1455 Bischof von Würzburg war.

Gottfried hatte fünf Geschwister. Die älteste Schwester Elisabeth (* 1467) heiratete in die Grafenfamilie von Helfenstein ein. Friedrich (* 1468) übernahm später die Herrschaft Speckfeld. Georg (* 1470) wurde Domherr in Würzburg und Bamberg und stieg zum Bischof von Bamberg (1505-1522) auf. Ludwig starb früh, und Susanna verschied schon kurz nach der Geburt im Juni 1475 zusammen mit der Mutter. Die nun verwaisten Kinder - Gottfried war gerade ein Jahr alt - wurden unter die Vormundschaft des einzigen Bruders des Schenken Georg gestellt, den Würzburger, Bamberger und Straßburger Domherrn Schenk Wilhelm. Dieser bestimmte und beeinflusste von nun an für mehrere Jahrzehnte entscheidend die Limpurger Politik und Geschicke.

Nachdem die Limpurger sich zuvor lange Zeit am kurfürstlichen Hof in Heidelberg orientiert hatten, wurde der junge Schenk Gottfried erstmalig an den Brandenburgischen Hof von Markgraf Friedrich in Ansbach vermittelt. Dort lernte er seine Frau, die adlige Margarethe Schlick, kennen, die er 1497 heiratete. Im selben Jahr teilte man das Limpurger Land, Friedrich erhielt den Speckfelder Landesteil, Gottfried die Herrschaft Limpurg.

Christoph Fröschel charakterisiert Schenk Gottfried wie folgt: von Natur ein frommer friedlicher sittsamer und freundlicher Herr ${ }^{8}$, Georg Salomon Ziegler, der Limpurgische Chronist in Markt Einersheim, bescheinigt ihm, dass er der Regierung gar löblich vorstand. Mit der Reichsstadt Hall stand er jedoch lange Zeit in feindlichen Auseinandersetzungen. Der Hällische Rat verbot seinen Bürgern, in Unterlimpurg Geld auszugeben und an die Schenkischen Untertanen Arbeitsaufträge irgendwelcher Art zu vergeben. 1514 vermittelte schließlich sein Bruder Georg, der Bamberger Bischof, einen Vergleich zwischen Hall und Limpurg über die Grenzen des Halsgerichts zu Limpurg, Jagdrechte, die Landhege und das Zollhaus Langenfeld. Im gleichen Jahr schlichtete der Markgraf Friedrich auf der Plassenburg einen Streit zwischen Schenk Gottfried und den Brüdern von Schlick wegen ausstehenden Heiratsguts seiner Frau Margaretha.

Als sein Bruder Friedrich 1521 ohne männliche Erben starb, fiel die Herrschaft Speckfeld an Schenk Gottfried. Er übergab diese bereits 1523 an seinen Sohn Karl. Der zweite Sohn, Erasmus, sollte die Herrschaft Limpurg erhalten. Der ist den Hallern wohlbekannt als Geliebter der Anna Büschler, die von etwa 1515 bis 1520 im Dienst der Schenkenfamilie stand. Die Schenkin, die Hermann Büschler mit dem Satz, seine Tochter brauche ihrem Sohn keine Hemden mehr anzumessen, dies könne sie selbst tun, alarmiert hatte, war Margarethe Gräfin von Schlick, die Frau Schenk Gottfrieds. 
Nach dem Tod seines Bruders Friedrich war Gottfried der Älteste des gesamten Limpurger Hauses und wurde 1521 auf dem Reichstag zu Worms mit den Regalien und Reichslehen und 1523 mit dem Erbschenkenamt belehnt.

Die Haller, so heißt es, hätten Schenk Gottfried den „Götz mit der leeren Tasche ${ }^{\text {"9 }}$ genannt. Dafür gab es Gründe. Bei der Landesteilung mit der Gaildorfer Linie der Schenken im Jahr 1481 musste deren Anteil an Speckfeld durch Ländertausch und eine Geldsumme von 4.200 Gulden abgelöst werden, wofür im folgenden Jahr die Herrschaft Adelmannsfelden verpfändet werden musste. Und durch die Limpurg-Speckfelder Landesteilung im Jahr 1497 waren die einträglicheren Speckfelder Besitzungen für die hiesigen Schenken weggefallen. Ihr Land war nun nicht mehr allzu groß. Auch werden die Haller nach dem Tod Schenk Georgs 1475 versucht haben, das Machtvakuum für ihre Belange zu nutzen. Zudem hatte die Erwerbung der Vogtei über die Comburg und deren Umwandlung in ein Chorherrenstift, bei der der Chorherr Schenk Wilhelm die zentrale Rolle spielte, Limpurg viel gekostet an Geld und Gütern. Kein Wunder, dass man nun auf laufende Kredite angewiesen war. So hat z. B. Schenk Wilhelm 1490 einen Kredit von 1.600 Gulden aufgenommen, von dem Schenk Gottfried noch 1513 den jährlichen Zins von 80 Gulden zu zahlen hatte und den erst Schenk Erasmus 1556 vollends zurückbezahlt hat ${ }^{10} .1522$ nimmt Schenk Gottfried bei Agatha Offnerin zu Hall 1.300 Gulden ${ }^{11}$, 1525 bei Philipp Keck zu Unterlimpurg 600 Gulden $^{12}$ Kapital auf. Schenk Erasmus versuchte später, das Problem zu lösen, indem er seine Stammburg Limpurg teuer an die Reichsstadt Hall verkaufte.

Eine andere Konstante während Gottfrieds Regierungszeit war die Auseinandersetzung mit dem Stift Comburg über den Limpurger Schirm. 1503 war zwar durch Bischof Lorenz zu Würzburg ein Vertrag zwischen Limpurg und Comburg über die Verleihung der Pfründen, die Jagd und die Gerichtsbarkeit des Stifts zustande gekommen, allein der Streit ging weiter. Schenk Gottfried versuchte, seinen Nutzen aus den Vogteirechten zu ziehen, die Stiftsherren fühlten sich von ihm bedrängt und wollten sich in ihre Politik nicht dreinreden lassen. In Würzburg liefen die gegenseitigen Anklagen ein. Schenk Gottfried drohte dem Würzburger Bischof damit, die limpurgischen Dörfer, die dem Bischof als Gegenleistung für die Übertragung der Vogtei über die Comburg als Lehen überlassen worden waren, wieder zurückzunehmen, wenn dem Treiben der Comburger Stiftsherren nicht gewehrt würde. Schließlich bestätigte der Bischof den Limpurger Schirm über die Comburg, doch die Auseinandersetzungen vor Ort

9 Gerd Wunder, Max Schefold, Herta Beutter: Die Schenken von Limpurg und ihr Land (FWFr

20). Sigmaringen 1982, S. 36.

10 StA Ludwigsburg B 113 I U 889 und Bü 1876.

11 Ebd. U 852.

12 Ebd. U 846. 
gingen weiter. Angeblich gab man darüber auf der Comburg sogar die Schenkenkapelle mit dem limpurgischen Erbbegräbnis dem Verfall preis ${ }^{13}$.

1519 verglich sich Bischof Georg von Bamberg, Gottfrieds Bruder, in Haßfurt erneut mit Bischof Konrad von Würzburg bezüglich des Schirms der Limpurger über die Comburg. Doch 1522 starb Bischof Georg in Bamberg, und das Tauziehen um die Macht auf der Comburg ging weiter.

Die Chorherren der Comburg machten Schenk Gottfried das Leben recht sauer, bis er solches auch darüber beschloss ${ }^{14}$, wie es Georg Salomon Ziegler formuliert.

Neben Karl und Erasmus und den Töchtern Anna und Sophia hatte Schenk Gottfried noch weitere vier Söhne. Drei von ihnen - nach Gottfried beziehungsweise Wilhelm Gottfried benannt, starben bald nach der Geburt und wurden - wie bei Johann Gottfried Biedermann ${ }^{15}$ überliefert ist - ebenfalls in der Unterlimpurger Kirche beigesetzt. Den jüngsten Sohn Philipp (*1515) konnte Schenk Gottfried mit einer Ausnahmegenehmigung bereits mit acht Jahren als Domherrn in Würzburg unterbringen. Auch in Bamberg sicherte er ihm eine Domherrenpfründe, und 1523 gelang es Schenk Gottfried sogar, den 13-Jährigen als Propst des Stifts Comburg durchzusetzen. Darüber kam es jedoch zum Streit mit Caspar Mein, dem Dekan von St. Stefan in Bamberg, der vom Papst Anwartsrechte auf die Propstei des Stifts Comburg erhalten hatte und nun mittels eines Vergleichs abgefunden werden musste ${ }^{16}$. Schenk Philipp lebte in Bamberg und Würzburg, ob er sich je auf der Comburg aufgehalten und betätigt hat, ist nicht belegt. Als er 1545 starb, wurde er in der Sepultur des Würzburger Doms beigesetzt.

In die Regierungszeit Schenk Gottfrieds fällt der Beginn der Reformation und der Bauernkrieg. Wenn sich auch die Reformation im Limpurger Land erst unter Schenk Erasmus allmählich durchsetzte, stand Schenk Gottfried der protestantischen Sache doch aufgeschlossen gegenüber ${ }^{17}$. Mehrfach setzte sich Schenk Gottfried mit Pfarrern seines Landes auseinander. So ließ er beispielsweise 1515 Lienhart Hauser, den Pfarrer in Sontheim, eines Bauern wegen gefangen auf die Limpurg führen. Auf Druck des Haller Kapitels mit einem Interdikt überantwortete er ihn an den Würzburger Bischof. 1528 veranlasste er die Einkerkerung und peinliche Befragung des Pfarrers Lorenz Reichlin von Michelbach. Den Einspruch Graf Albrechts von Hohenlohe, der das Patronatsrecht in Michelbach innehatte, wies Schenk Gottfried mit der Begründung ab, es handle sich um „öffentlich verwirkte Sachen in baurischer Empörung“, die in seine Zuständigkeit

13 Wie Anm. 1.

14 Ebd.

15 Wie Anm. 4.

16 StA Ludwigsburg B 113 I U 662.

17 Reinhold Hohl: Unsere Kirche erzählt aus der Geschichte unserer Gemeinde. In: Michelbach an der Bilz. Beiträge zu Geschichte und Gegenwart. Hg. Gemeinde Michelbach/Bilz. 1980. S. $161 \mathrm{f}$. 
fielen. Auf Bitten von Freunden und Verwandten ließ er Lorenz Reichlin gegen eine Geldstrafe schließlich wieder frei ${ }^{18}$.

1528 einigte sich Schenk Gottfried noch mit der Reichsstadt Hall auf einen Vertrag über Lehengüter, Wehre und die Landheg.

Am 9. April 1530, in seinem 56. Lebensjahr, starb Schenk Gottfried.

18 Wie Anm. 17, S. 160. 